\title{
CHROMOSOME NUMBERS OF SUCCULENT PLANTS
}

\author{
BRIAN SNOAD \\ John Innes Horticultural Institution, Bayfordbury, Hertford, Herts
}

Received $3^{1 \text {.i. } 5^{\mathrm{I}}}$

The following lists of chromosome numbers have been compiled from the collection of succulent plants established at the John Innes Horticultural Institution by G. D. Rowley, to whom I am grateful for help in the identification of certain species and for enlarging the collection for cytological studies.

The majority of observations were made from root-tip squashes pretreated with $a$-bromonaphthalene, fixed in acetic alcohol and stained in Feulgen, few flowers being available for the study of meiosis.

Two main groups were studied,
(a) Aloine-3 genera.
(b) Ficoidacea-33 genera.

With one exception-noted below-the plants are all South African in distribution.

The basic number of the Ficoidacee is 9. The chromosomes are small and all with median centromeres, there being no differentiation of types.

The basic number of the Aloine is 7, and here there is far more differentiation between the chromosomes. They can be split up into four groups A, B, C and D as shown in fig. I (page 280).

There are slight differences between the karyotypes of the three genera-mainly differences involving the position of the satellites. When satellites are present in Haworthia they are similar in position to those in Aloë. Resende (1937), however, reported that the number of satellites is not constant for all the species in certain genera in the Aloina.

The degrees of polyploidy in both the Aloina and the Ficoidacee are summarised in table $\mathrm{I}$.

TABLE I

\begin{tabular}{|c|c|c|c|c|c|c|c|c|}
\hline & & $2 x$ & $3^{x}$ & $4^{x}$ & $5^{x}$ & $6 x$ & $7 x$ & $8 x$ \\
\hline Ficoidacee & . & $148(5 \mathrm{I})$ & $7(4)$ & 53 (12) & & $3(2)$ & $\cdots$ & I (I) \\
\hline Alö̈ : & . & 82 (I5) & $\dddot{1}$ & $3(1)$ & $I(\ldots)$ & $4(4)$ & $\cdots$ & $\cdots$ \\
\hline $\begin{array}{l}\text { Gasteria } \\
\text { Haworthia }\end{array}$ & . & $\begin{array}{l}23 \text { (II) } \\
75(35)\end{array}$ & $5(2)$ & $\begin{array}{r}3(\ldots) \\
24(5)\end{array}$ & $5(2)$ & Io (3) & $I(\ldots)$ & $2(\mathrm{I})$ \\
\hline \multicolumn{2}{|c|}{ Total (Aloë excepted) } & 246 & 13 & 80 & 5 & $r_{3}$ & I & 3 \\
\hline
\end{tabular}

(The numbers in brackets refer to the 148 individuals reported in the present list of chromosome numbers.)

It will be noticed that apart from Aloë the even-numbered species fall into geometrical progressions and the odd-numbered species may be regarded as a function of the numbers above and below each. 
If these are $a$ and $b$ then the value $\mathrm{x} / 9 \sqrt{a \bar{b}}$ roughly fits. The odd numbers are therefore presumably hybrids between species above and below them in polyploidy.

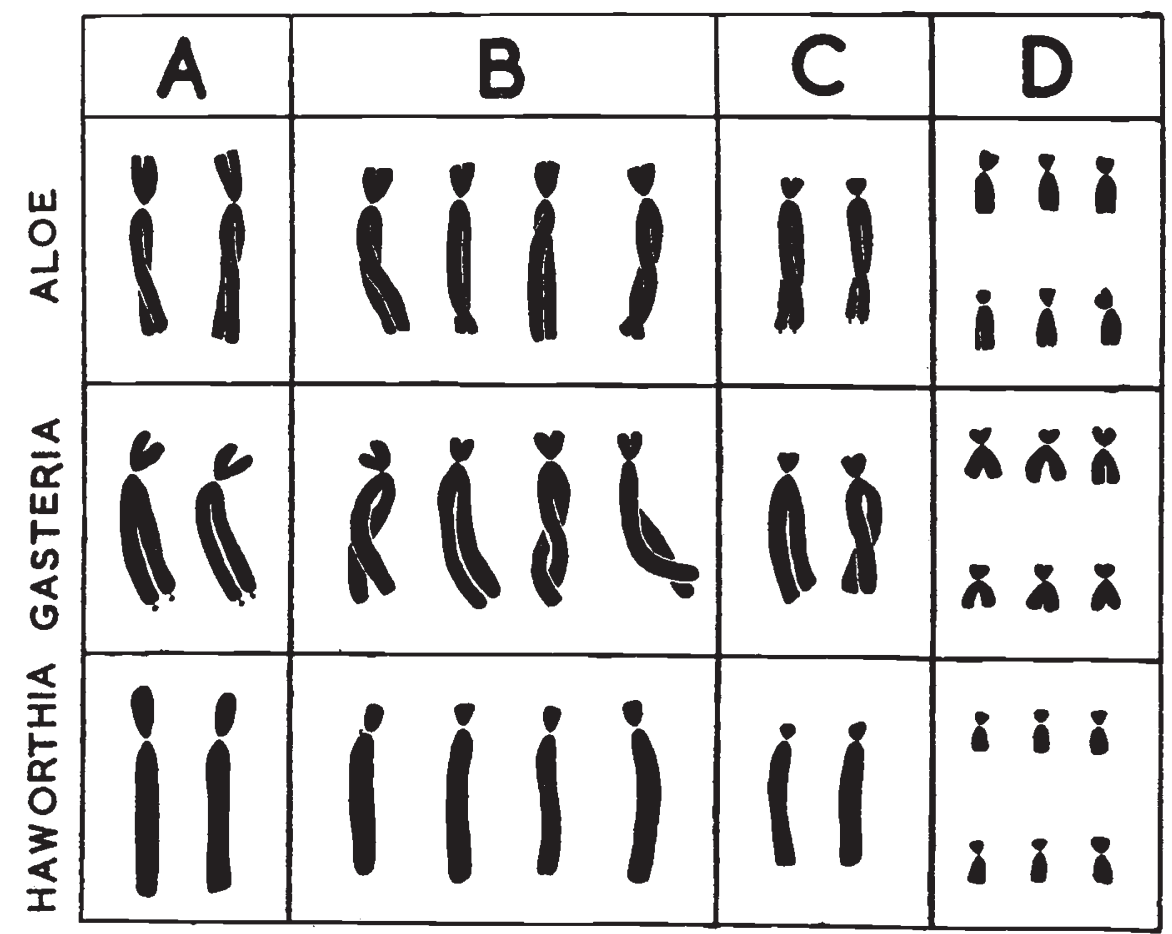

FIG.

In conclusion it should be pointed out that no "chromatic agglutination" as referred to by other workers (Resende, Rijo and Pinto-Lopes) was seen in any normal preparations of the Aloine. However, stickiness was easily induced by over-hydrolysis of the material prior to Feulgen staining and this may have been the cause of the trouble.

ALOË $2 x$
arborescens Miller
aristata Schult.
brevifolia Miller
coccinea ?
commixta Berger
greenii Baker
longifolia Haw.
plicatilis Miller
salaris Hort.
saponaria Haw.
schimperi $*$ Todaro
sp. (J. Brown, Southall)
spinosissima Hort.
striatula Haw.
variegata Linn.

$$
\text { ALOINAE : } x=7
$$

ciliaris Haw. (Edinburgh)

ciliaris Haw. (J. Brown)

ciliaris Haw. (Monaco)

ciliaris Haw. (V. Higgins $\dagger$ )

ciliaris Haw., tidmarshii Schönl.

tenuifolia Lam.

tenuior Haw.

* Abyssinian.

$\dagger$ Parent plant and r950 seedling. 


\section{ALOINAE-continued}

\section{GASTERIA}

armstrongii Schönl.

bayfieldii Baker

beckeri Schönl.

carinata Duval

colubrina N. E. Brown

croucheri Baker

\section{HAWORTHIA}

angusta?

aristata Haw.

aspera Haw.

atrovirens Haw.

attenuata Haw.

beanii G. G. Smith

beanii G. G. Smith, minor G. G. S.

coarctata Haw.

correcta v. Poelln.

cuspidata Haw.

cymbiformis Duval

fulva G. G. S.

glabrata Baker

herrei v. Poelln.

herrei v. Poelln., depauperata v. Poelln.

hilliana v. Poelln.

icosiphylla Baker

krausiana?

lepida G. G. S.

maughanii v. Poelln.

mucronata Haw., polyphylla?

perplexa v. Poelln.

planifolia Haw.

pumila Duval

ramosa G. G. S.

reinwardtii Haw., brevicula G. G. S.

reinwardtii Haw., grandicula G. G. S.

reinwardtii Haw., kaffirdriftensis, G. G. S.

reinwardtii Haw., riebeckensis G. G. S.

subregularis Baker

syringoidea?
$2 x$

levis Haw.

liliputana v. Poelln.

pulchra Haw.

rurex Hort.

$s p$. Hort.

tortuosa Haw., var.

truncata Schönl.

turgida Haw.

viscosa Haw.

reinwardtii Haw., chalumnensis G. G. S. reinwardtii Haw., peddiensis G. G. S.

$4^{x}$

glauca Baker

greenii Baker, silvicola G. G. S.

reinwardtii Haw., committeesensis, G. G.S. reinwardtii Haw., huntsdriftensis G. G. S. reinwardtii Haw., valida G. G. S.

$5^{x}$

rubrobrunea v. Poelln.

sampaiana Res., brotereana Res.

sampaiana Res.

$$
5^{x+1}
$$

$6 x$

coarctata Haw., v. haworthii Res., , v. kraussii Res.

coarctatoides Res.

tessellata Haw.

FICOIDACE E : $x=9$

(i) $2 x$ Genera

APTENIA

cordifolia Schwant.

ARGYRODERMA

ovale L. Bol.

ARIDARIA

canaliculata L. Bol.

tetragona L. Bol.

ASTRIDIA

maxima Schwant.

BIJLIA

cana N. E. Br.
CARPOBROTUS edulis N. E. Br. fourcadei $\mathrm{L}$. Bol.

CARRUANTHUS caninus Schwant.

CEROCHLAMYS pachyphylla L. Bol. CONICOSIA

capensis N. E. Br. communis N. E. Br. pugioniformis N. E. Br. 


\section{FICOIDACEAE-continued}

(1) $2 x$ Genera-continued

CORPUSCULARIA

lehmannii Schwant.

CYLINDROPHYLLUM

calamiforme Schwant.

DINTERANTHUS

microspermus Schwant.

ECHINUS

maximiliani $\mathrm{N}$. E. Br.

EREPSIA

inclaudens N. E. Br.

HEREROA

incurva L. Bol.

stanfordic $\mathrm{L}$. Bol.

NANANTHUS

aloides N. E. Br.

crassipes L. Bol.

\author{
ODONTOPHORUS \\ marlothii N. E. Br. \\ PLEIOSPILOS \\ prismaticus Schwant. \\ PRENIA \\ relaxata N. E. Br. \\ PSILOCAULON \\ granulicaule N. E. Br. \\ RHINEPHYLLUM \\ comptonii L. Bol. \\ STOMATIUM \\ conradii? \\ paucidens L. Bol. \\ rosei ?
}

(ii) $4 x$ Genera

\section{DROSANTHEMUM \\ candens Schwant.} purpureus Graessn.

(iii) Mixed Genera

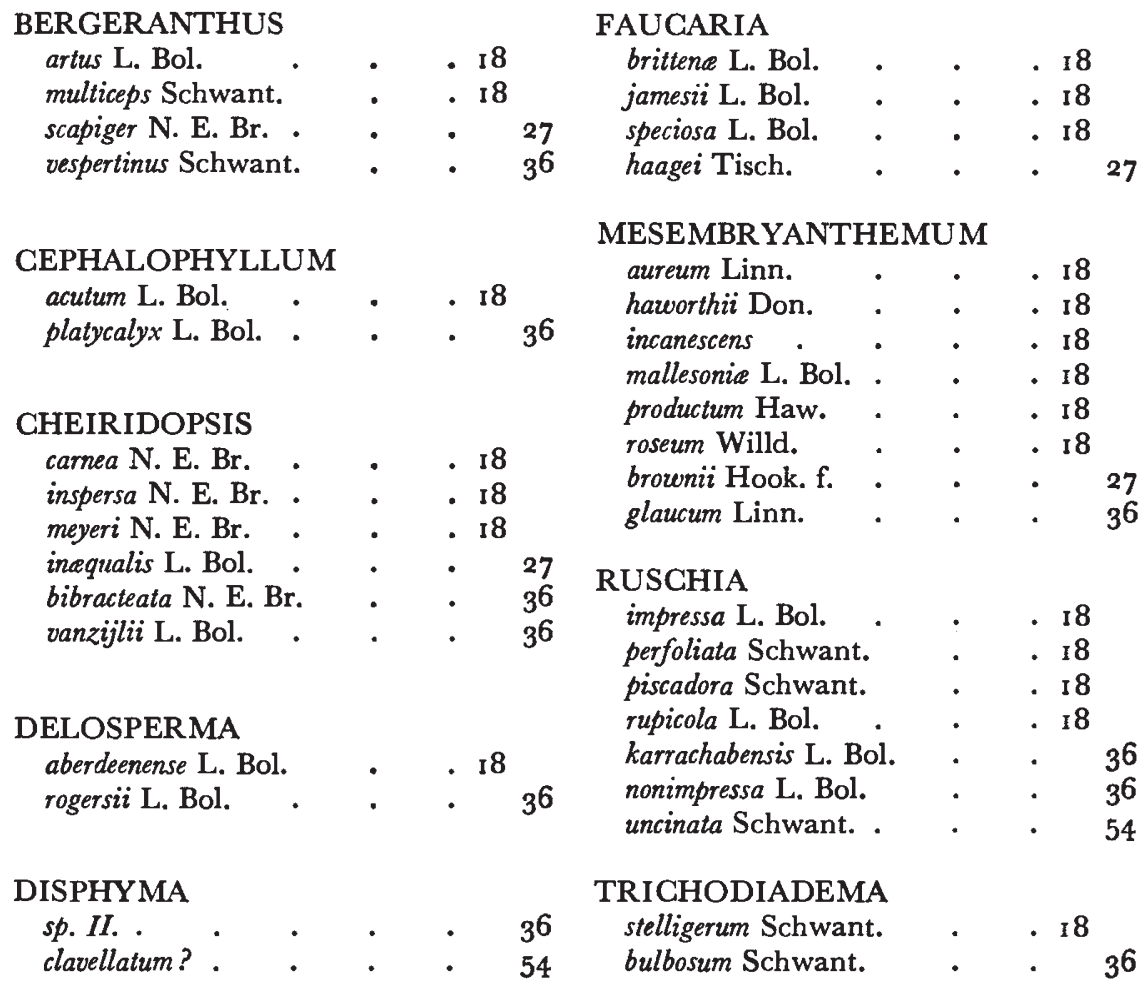




\section{REFERENCES}

Pinto-Lopes, J. 1946. Cariological studies of the Aloine IV. Portug. Acta Biol., I, I 87-234.

RESENDE, F. I937. Ueber die Ubiquitaet der Sat. chromosomen bei den bluetenpflanzen. Planta, 26, 757-805.

RESENDE, F., AND RIJO, L. I 948 . Structure of chromosomes as observed in root-tips. Portug. Acta Biol., 2, I I 7-1 48 . 